\title{
Síntesis y Refinado por el Método Rietveld de la fase perovsquita obtenida a partir del Método de Co-precipitación vía Oxalato
}

\author{
Andarair G. dos Santos*, Francisco A. O. Fontes, João F. de Sousa y Carlson P. de Souza \\ Universidad Federal de Río Grande del Norte, Programa de Posgrado en Ingeniería Química, \\ Avenida Salgado Filho 3.000, Lagoa Nova - 59078-970 - Natal - RN - Brasil \\ (e-mail: andaraireq@yahoo.com.br, carlson@ufrnet.br) \\ *autor a quien dirigir la correspondencia
}

Recibido Abr. 06, 2010; Aceptado Feb. 15, 2010; Versión Final recibida May. 27, 2010

\begin{abstract}
Resumen
Material cerámico del tipo perovisquita $\mathrm{SrCo}_{0,8} \mathrm{Fe}_{0,2} \mathrm{O}_{3-\delta}$ fue sintetizado usando el método de coprecipitación en medio oxalato. Durante los análisis térmico-gravimétrico y térmico-diferencial (TG/DTA) se verificó que independientemente de la naturaleza del reactivo o agente precipitante adoptado en la síntesis del precursor, la curva termogravimétrica presenta tres etapas, con pérdida de masa total de aproximadamente el $40 \%$. Mediante difracción de rayos X se verificó que el tamaño medio del cristalito fue de aproximadamente $77,22 \mathrm{~nm}$ utilizando nitrato de Hierro III, casi un $83 \%$ superior al obtenido usando oxalato de Hierro III $(42,23 \mathrm{~nm})$. Sin embargo, la variación del agente precipitante no afectó prácticamente al tamaño mediano de cristalino (42,95 $\mathrm{nm}$ y $42,23 \mathrm{~nm}$ ). El material presenta isotermas de tipo II, con una superficie específica de aproximadamente $4,41 \mathrm{~m}^{2} \mathrm{~g}^{-1}$.
\end{abstract}

\section{Synthesis and Refining by Rietveld Method of the perovskite phase obtained using Oxalate Co- precipitation Method}

\begin{abstract}
Powders of perovskite $\mathrm{SrCo}_{0.8} \mathrm{Fe}_{0.2} \mathrm{O}_{3-\delta}$ were synthesized using oxalate co-precipitation method. According to the thermal gravimetry and differential thermal analysis (TG/DTA), total mass loss was $40 \%$ corresponding to three different steps and the precipitant agent as well the starting chemical elements have no influence on the process. Perovskite phase was identified by $\mathrm{x}$-ray diffraction and the average crystallite size was $77.22 \mathrm{~nm}$ using iron nitrate (III) as starting material while $42.23 \mathrm{~nm}$ were obtained using iron oxalate (III). Ammonium oxalate and equimolar mixtures of ammonium oxalate and oxalic acid were used as precipitant agents and the results shown that these reactants have no influence on the average crystallite size $(42.95 \mathrm{~nm}$ and $42.23 \mathrm{~nm})$. The material presents isotherms of type II, with especific surface of approximately $4,41 \mathrm{~m}^{2} \mathrm{~g}^{-1}$.
\end{abstract}

Keywords: perovskite, co-precipitation method, oxalate, refining Rietveld method 


\section{INTRODUCCIÓN}

La sinergia existente entre desarrollo tecnológico, necesidades económicas y factores ambientales está causando un gran cambio en el uso de los materiales, en el sentido de substituir materiales tradicionales de la industria, sobre todo los metales, por otros materiales, como los plásticos, las cerámicas y sus composites, con prestaciones eficaces y a bajo coste.

En función de las características intrínsecas de los materiales cerámicos, como: baja conductividad térmica, alta resistencia a la corrosión y a la abrasión y capacidad de soportar altas temperaturas sin deformarse, además de otras características específicas presentes en algunos de esos materiales, como la conductividad iónica y eléctrica, su uso está creciendo de forma sorprendente en una infinidad de aplicaciones industriales.

Las membranas cerámicas inorgánicas están recibiendo en las últimas décadas una mayor atención en su investigaciones y desarrollo, al presentar alta conductividad mixta (iónica y eléctrica) y permeabilidad de oxígeno (Teraoka et al., 1985, 1988; Shao et al., 2001; Mineshige et al., 2005; Mcintosh et al., 2006). Esas membranas cerámicas son materiales prometedores para muchos procesos industriales que requieren un suministro continúo de oxígeno puro, como en procesos Fisher-Trospsh y síntesis de metanol. Sin embargo, cuando esos materiales son sometidos la altas temperaturas pueden presentarse cambios de fase o estructurales. Los cambios estructurales pueden también se inducidos por los ciclos repetidos de sorción y desorción de la sustancia que permea (por ejemplo, oxígeno) pudiendo terminar en fractura del material.

En esta situación, se están realizando muchos estudios tratando de minimizar los problemas que tienen estos materiales y teniendo como objetivo final la búsqueda de un material, con capacidades de síntesis y/u operación reactiva que asocie las propiedades de alta permeabilidad de oxígeno y alta estabilidad termodinámica en un único material.

El método de preparación de los materiales influye posiblemente en las características fisicoquímicas de la membrana, como: su microestrutura su cristalinidad, y su uniformidad de la composición local, mejorando probablemente la propiedad de permeabilidad de oxígeno (Ikeguchi et al., 2003).

Podemos utilizar varios métodos de síntesis de síntesis para la obtención de materiales cerámicos. La mayoría de las síntesis industriales de polvos para aplicación en cerámicas se obtiene por medio del método cerámico tradicional o reacción en estado sólido, también llamada de mezcla de óxidos (Yang et al., 2003; Ikeguche et al., 2005; Casanova et al., 2004; Liu et al., 2000; Teraoka et al., 2002). Entre los varios métodos de síntesis encontrados en la literatura, podemos citar: evaporation by dryness (Teraoka et al., 1988; Yaremchenlo y Kharton, 1999), método Pechini (Waller et al., 1996;), sol-gel (Vargas et al., 2007), técnicas de los citratos (Shao et al., 2000; Dong et al., 2001; Tong et al., 2002), creación de complejo combinando EDTA-citrato (Shao et al., 2001; Ikeguchi et al., 2003; Lu et al.; 2005; 2006), co-precipitación (Oliveira et al.; 2007; Rivas et al., 2008; Ouaguenouni et al., 2009; Tanabe Y Assaf, 2009).

El control cuidadoso del método utilizado para sintetizar los materiales es de importancia fundamental, ya que no sólo van a influir fuertemente en las características de los materiales en el proceso sino también en las reacciones que ocurren durante la calcinación, que son las que van a determinar las propiedades finales. Para la mayoría de las aplicaciones es necesaria, la síntesis controlada de alta pureza con el fin de obtener propiedades repetibles. La elección del método es de importancia fundamental para controlar mejor el área superficial, el tamaño de las partículas y su bajo grado de aglomeración.

Todas estas técnicas tienen sus ventajas y desventajas, debido a la diferencia del principio químico utilizado con cada tecnología. El método de co-precipitación ofrece algunas ventajas, como: preparación simple y rápida, fácil control del tamaño de partícula y composición y varias posibilidades de controlar el estado de la superficie total de las partículas y su homogeneidad. 
De acuerdo con la literatura, las membranas ternaria y cuaternaria $\mathrm{SrCo}_{0,8} \mathrm{Fe}_{0,2} \mathrm{O}_{3-\delta}$ y $\mathrm{Ba}_{0,5} \mathrm{Sr}_{0,5} \mathrm{Co}_{0,8} \mathrm{Fe}_{0,2} \mathrm{O}_{3-\delta}$ poseen alto flujo de permeación de oxígeno. $\mathrm{El} \mathrm{La}_{0,2} \mathrm{Sr}_{0,8} \mathrm{Co}_{0,2} \mathrm{Fe}_{0,8} \mathrm{O}_{3-\delta}$ y el $\mathrm{La}_{0,2} \mathrm{Ba}_{0,8} \mathrm{Co}_{0,2} \mathrm{Fe}_{0,8} \mathrm{O}_{3-\delta}$ son dos materiales cerámicos aplicados intensivamente en separación de $\mathrm{O}_{2}$ y en reactores de membrana de oxidación parcial de metano (POM) para generación de gas de síntesis. Con base en estas informaciones decidimos partir de la síntesis de el material $\mathrm{SrCo}_{0,8} \mathrm{Fe}_{0,2} \mathrm{O}_{3-\delta}$ y realizar un estudio de un método de síntesis basado en el método de coprecipitación de oxalato, en un reactor de mezcla con agitación, como método de síntesis alternativo para producción de los polvos cerámicos de mezcla conductora de óxidos y con el objetivo de obtener polvos con tamaño en el orden de la escala nanométrica que según la literatura alcanzan mayores flujos de permeación de oxígeno en reactores de membrana para producción de gas de síntesis.

En el presente trabajo el material cerámico de tipo perovisquita $\mathrm{SrCo}_{0,8} \mathrm{Fe}_{0,2} \mathrm{O}_{3-\delta}$ se sintetizó con base en el método de co-precipitación en medio oxalato, teniendo como objetivo principal el estudio del camino de síntesis más simple y con mayor rendimiento, permitiendo la obtención de polvos con tamaño mediano de cristalito de orden nanometrico. También han sido evaluados la influencia de algunos parámetros de síntesis, como: el agente precipitante y la naturaleza de los reactivos empleados en la síntesis.

\section{EXPERIMENTAL}

\section{Reactivos utilizados}

La síntesis del material $\mathrm{SrCo}_{0,8} \mathrm{Fe}_{0,2} \mathrm{O}_{3-\delta}$ se realiza basándonos en el método de co-precipitación en medio oxalato sean usado los siguientes reactivos: carbonato de estroncio $\left(\mathrm{SrCO}_{3}\right)$ (Aldrick, 99,99 $\%$ ), oxalato de hierro hexahidratado $\left(\mathrm{Fe}\left(\mathrm{C}_{2} \mathrm{O}_{4}\right)_{3} 6 \mathrm{H}_{2} \mathrm{O}\right)$ (Aldrick, $98 \%$ ), nitrato de hierro nanohidratado $\left(\mathrm{Fe}\left(\mathrm{NO}_{3}\right)_{3} 9 \mathrm{H}_{2} \mathrm{O}\right)$ (Aldrick, $\left.98+\%\right)$, nitrato de cobalto hexahidratado $\left(\mathrm{Co}\left(\mathrm{NO}_{3}\right)_{2} 6 \mathrm{H}_{2} \mathrm{O}\right)$ ([Aldrick], $98 \%$ ), hidróxido de amonio $\left(\mathrm{NH}_{4} \mathrm{OH}\right)$ (Fluka, $25 \%$ ), trietilamina $\left(\mathrm{C}_{6} \mathrm{H}_{15} \mathrm{~N}\right.$ ) (Aldrick, $99 \%$ ), acido oxálico $\left(\mathrm{H}_{2} \mathrm{C}_{2} \mathrm{O}_{4} 2 \mathrm{H}_{2} \mathrm{O}\right)$ (Aldrick, $99 \%$ ), oxalato de amonio $\left(\left(\mathrm{NH}_{4}\right)_{2} \mathrm{C}_{2} \mathrm{O}_{4} \mathrm{H}_{2} \mathrm{O}\right)$ (Aldrick, $\left.99 \%\right)$ y agua desionizada.

Se usaron como agentes precipitantes: una mezcla equimolar de solución de acido oxálico y oxalato de amonio en razón másica (1:1) y oxalato de amonio. Para control del pH usamos trietilamina. La tabla 1 resume las condiciones experimentales adoptadas paras síntesis del material.

Tabla 1: Condiciones experimentales adoptadas para síntesis de los precursores utilizando diferentes reactivos y agentes precipitantes con base en el método vía co-precipitación en medio oxalato

\begin{tabular}{|c|c|c|}
\hline Experimento & Agente precipitante & Reactivos \\
\hline Muestra 1 & $\begin{array}{c}\text { Trietilamina } \\
\left(\left(\mathrm{NH}_{4}\right)_{2} \mathrm{C}_{2} \mathrm{O}_{4} \mathrm{H}_{2} \mathrm{O} / \mathrm{H}_{2} \mathrm{C}_{2} \mathrm{O}_{4} 2 \mathrm{H}_{2} \mathrm{O}(1: 1)\right.\end{array}$ & $\begin{array}{c}\mathrm{SrCO}_{3} \\
\mathrm{Co}\left(\mathrm{NO}_{3}\right)_{2} 6 \mathrm{H}_{2} \mathrm{O} \\
\mathrm{Fe}\left(\mathrm{NO}_{3}\right)_{3} 6 \mathrm{H}_{2} \mathrm{O}\end{array}$ \\
\hline Muestra 2 & $\begin{array}{c}\text { Trietilamina } \\
\left(\mathrm{NH}_{4}\right)_{2} \mathrm{C}_{2} \mathrm{O}_{4} \mathrm{H}_{2} \mathrm{O}\end{array}$ & $\begin{array}{c}\mathrm{SrCO}_{3} \\
\mathrm{Co}\left(\mathrm{NO}_{3}\right)_{2} 6 \mathrm{H}_{2} \mathrm{O} \\
\mathrm{Fe}_{2}\left(\mathrm{C}_{2} \mathrm{O}_{4}\right)_{3} 6 \mathrm{H}_{2} \mathrm{O}\end{array}$ \\
\hline Muestra 3 & $\begin{array}{c}\text { Trietilamina } \\
\left(\mathrm{NH}_{4}\right)_{2} \mathrm{C}_{2} \mathrm{O}_{4} \mathrm{H}_{2} \mathrm{O} / \mathrm{H}_{2} \mathrm{C}_{2} \mathrm{O}_{4} 2 \mathrm{H}_{2} \mathrm{O}(1: 1)\end{array}$ & $\begin{array}{c}\mathrm{SrCO}_{3} \\
\mathrm{Co}\left(\mathrm{NO}_{3}\right)_{2} 6 \mathrm{H}_{2} \mathrm{O} \\
\mathrm{Fe}_{2}\left(\mathrm{C}_{2} \mathrm{O}_{4}\right)_{3} 6 \mathrm{H}_{2} \mathrm{O}\end{array}$ \\
\hline
\end{tabular}

\section{Procedimiento experimental}

Para la síntesis de los materiales utilizamos un reactor de mezcla fabricado en vidrio montado en un sistema modular, compuesto por un motor/eje de agitación controlada por inversor de frecuencia (CFW-08/[WEQ]). 
Se añadió inicialmente un volumen de $200 \mathrm{~mL}$ del agua desionizada en el reactor. Inmediatamente, la solución base con los elementos que componen la composición del material ( $\mathrm{Sr}$, Co y $\mathrm{Fe}$ ) juntamente con el agente precipitante y la [trietilamina] se añaden por goteo simultaneo en el reactor, bajo agitación continúa y a temperatura ambiente mantenida durante todo proceso de síntesis. Se usó trietilamina para control del $\mathrm{pH}(\sim 11)$ de la mezcla reactiva.

El precipitado fue lavado varias veces con agua destilada y después filtrado en un sistema de filtro tipo miliporoso acoplado a una bomba de vacío (TE-058 TECNAL). Después secados en (estufa EDG) y por último, molidos en almirez de ágata.

La formación de la fase se acompañó con análisis térmicos utilizando una balanza termogravimétrica (SETARAM TG - DTA 92). Utilizamos un crisol cerámico para análisis térmicos en atmósfera de aire en una franja de temperatura de 25 - $950{ }^{\circ} \mathrm{C}$, con tasa de calentamiento/enfriamiento de $5{ }^{\circ} \mathrm{Cmin}^{-1}$. Los resultados se presentan en forma de curva termogravimétrica (TG) y termodiferencial (DTA). Por último, las muestras fueron sometidas a tratamiento térmico, molidas y caracterizadas por Difracción de Rayos $-X$ / refinado por el método Rietveld $Y$ la morfologia de los polvos ceramicos se observó de microscopia eletronica de barrido (MEV). Microscópio Philips XL 30 ESEM.

Se realizaron análisis por difracción de Rayos - $X$ usando el método del polvo (Difractómetro de Rayos - X modelo Siemens Diffractometer), utilizando radiación CuKa y longitud de onda $\lambda$ igual a $1,5406 \AA$, con el fin de identificar las fases cristalinas presentes. Los diafragmas se compararon rayo $x$ se compararon con las normas JCPDS (número JCPDS: 79022 - ICSD).

A partir de los difractogramas realizamos un refinado por el método Rietveld para determinar el tamaño medio del cristalito de los materiales obtenidos y referirlos a las alteraciones en los parámetros de síntesis, basándonos en los picos de difracción de los planos y en la ecuación de Scherrer (Snyder, 1992). El método de Rietveld (Young, 1995) es un método de analisis de perfeccionamiento de estructuras cristalinas en el que todos los factores de contribuiciones a las intensidades pueden ser a la vez refinado hasta que la diferencia entre las muestras experimentales se calcula y reducir al mínimo (Wilson, 1995; Gualtieri et al, 2006, Martinez et al., 2006).

El programa DBWS 2.16 utiliza el algoritmo de Newton - Raphson para refinar y cuantificar estas intensidades medidas. El ajuste de los picos del difractograma se lleva a cabo con el perfil de la función Pseudo - Voigt que contiene modificada que contienen mezclas de las contribuiciones de Lorentziana y Gaussiana (Young, 1995).

Las analisis de superficie especifica y las isotermas de adsorción de punto de venta se obtuvieron por adsorción de nitrogeno en el material, tras el modelo propuesto por BET (Brunauer, Emmeett y Teller). El método BET considera el volumen de nitrógeno absorvido en la superficie de los sólidos por interacciones físicas a diversas presiones relativas en la temperatura del nitrógeno liquido a una presión de hasta $2 \mathrm{~atm}$ y la presión relativa $(\mathrm{P} / \mathrm{Po})$ de menos de 0,3 a bajas temperatura e presión.

Para esta prueba, se utilizó un medidor de área especifica metro Quanta Chrome NOVA - 1200E de superfície, y el analizador de pore, equipado con el sofware para determnar la superfície específica. El diámetro y volumen de poro se obtuvieron por el Barret - Joyner - Halenda (BJH). Las muestras fueron degasificadas durante 3 horas a $300^{\circ} \mathrm{C}$ para eliminar cualquier material fisissorvido dentro de los poros y la superficie del material.

La superficie y el tamaño promedio de partículas se determina a partir de la relación geométrica dada en la ecuación 4.

$$
D_{\mathrm{BET}}=6 / \mathrm{S}_{\mathrm{BET}} \rho
$$

$D_{B E T}$ donde es el diámetro medio de partículas, $S_{B E T}$ es área superficial específica y $\rho$ es la densidad teórica. 


\section{RESULTADOS Y DISCUSIÓN}

Análisis termogravimétrico y termodiferencial (TG - DTA)

Los materiales de composición $\mathrm{SrCo}_{0,8} \mathrm{Fe}_{0,2} \mathrm{O}_{3-\delta}$ del tipo perovsquita se sintetiza con base en el método vía co-precipitación en medio oxalato en tres condiciones distintas de síntesis, conforme a la Tabla 1.

La Figura 1 ilustra las curvas de termogravimentria (TG) (a) y termodiferencial (DTA) (b) para las muestras del precursor del $\mathrm{SrCo}_{0,8} \mathrm{Fe}_{0,2} \mathrm{O}_{3-\delta}$.
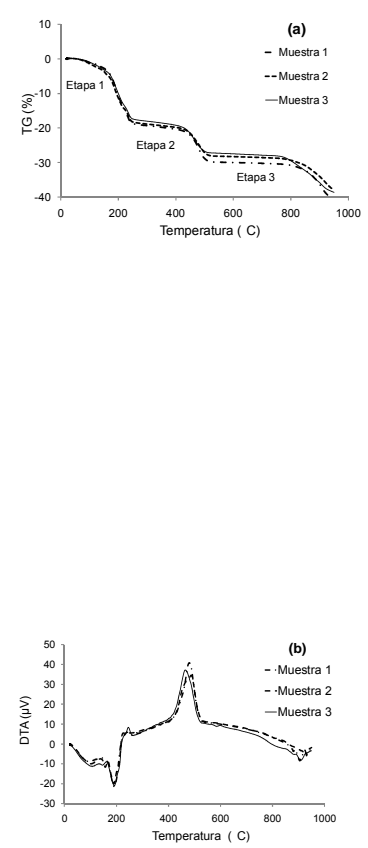

Fig.1: (TG) (a) y DTA (b) de las muestras del precursor óxido $\mathrm{SrCo}_{0,8} \mathrm{Fe}_{0,2} \mathrm{O}_{3-\delta}$ referido a las condiciones de síntesis: Muestras $(1-3)$. Tasa de calentamiento y enfriamiento: $5^{\circ} \mathrm{Cmin}^{-1}$

Las muestras de $\mathrm{SrCo}_{0,8} \mathrm{Fe}_{0,2} \mathrm{O}_{3-\delta}$ presentan tres etapas de descomposición térmica. La primera etapa es referente la deshidratación del material, con un pico endotérmico en la curva DTA alrededor de los $160^{\circ} \mathrm{C}$, conforme a la reacción (1). La segunda pérdida de masa se corresponde a la formación de $\mathrm{CO}$, y se asocia a un pico exotérmico a $480^{\circ} \mathrm{C}$, de acuerdo con a la reacción (2), con la formación del oxi-carbonato mixto de $\mathrm{SrCo}_{0,8} \mathrm{Fe}_{0,2} \mathrm{O}_{1,1} \mathrm{CO}_{3}$ según la reacción global (3). La última pérdida de masa se asocia a un pico endotérmico en el entorno de los $905{ }^{\circ} \mathrm{C}$ correspondiente posiblemente a la pérdida de $\mathrm{CO}_{2}$, con formación de óxido mixto $\mathrm{SrCo}_{0,8} \mathrm{Fe}_{0,2} \mathrm{O}_{3-\delta}$ según la reacción (4). Que de cuerdo con los resultados presentados por Qui et al., 1995, la fase $\mathrm{SrCo}_{0,8} \mathrm{Fe}_{0,2} \mathrm{O}_{3-\delta}$ se reproduce por encima de $770{ }^{\circ} \mathrm{C}$. Sin embargo, $800{ }^{\circ} \mathrm{C}$, hay la presencia de etapas secundarias, y probablemente $\mathrm{SrCO}_{3} \mathrm{Sr}_{6} \mathrm{Co}_{5} \mathrm{O}_{15}$, presentando monofásicos $\left(\mathrm{SrCo}_{0,8} \mathrm{Fe}_{0,2} \mathrm{O}_{3}\right.$ §) la temperatura de calcinación igaul a $950^{\circ} \mathrm{C}$. Los datos del análisis térmico se presentan en la Tabla 2. 
$\mathrm{Sr}\left[\mathrm{Co}_{0,8} \mathrm{Fe}_{0,2} \mathrm{O}_{1,1}\left(\mathrm{C}_{2} \mathrm{O}_{4}\right)\right], 3 \mathrm{H}_{2} \mathrm{O} \rightarrow \mathrm{Sr}\left[\mathrm{Co}_{0,8} \mathrm{Fe}_{0,2} \mathrm{O}_{1,1}\left(\mathrm{C}_{2} \mathrm{O}_{4}\right)\right]+3 \mathrm{H}_{2} \mathrm{O}$

$\mathrm{CO}+1 / 2 \mathrm{O}_{2} \rightarrow \mathrm{CO}_{2}$

$\mathrm{Sr}\left[\mathrm{Co}_{0,8} \mathrm{Fe}_{0,2} \mathrm{O}_{1,1}\left(\mathrm{C}_{2} \mathrm{O}_{4}\right)\right] \rightarrow \mathrm{Sr}\left[\mathrm{Co}_{0,8} \mathrm{Fe}_{0,2} \mathrm{O}_{1,1}\left(\mathrm{CO}_{3}\right)\right]+\mathrm{CO}$

$\mathrm{Sr}\left[\mathrm{Co}_{0,8} \mathrm{Fe}_{0,2} \mathrm{O}_{1,1}\left(\mathrm{CO}_{3}\right)\right] \rightarrow \mathrm{SrCo}_{0,8} \mathrm{Fe}_{0,2} \mathrm{O}_{2,1}+\mathrm{CO}_{2}$

De acuerdo a los análisis de TG y DTA de las muestras presentadas se puede verificar que independientemente de la naturaleza del reactivo o agente precipitante adoptado en la síntesis del precursor del material $\mathrm{SrCo}_{0,8} \mathrm{Fe}_{0,2} \mathrm{O}_{3-\delta}$ con base en el método de co-precipitación vía oxalato, la curva termogravimétrica se compone de tres etapas distintas de descomposición del material, con una pérdida de masa total de cerca del $40 \%$.

Tabla 2: Dados de las curvas de análisis térmico del precursor del $\mathrm{SrCo}_{0,8} \mathrm{Fe}_{0,2} \mathrm{O}_{3-\delta}$

\begin{tabular}{|c|c|c|c|c|}
\hline Muestras & Etapas & $\begin{array}{c}\text { Temperatura, } \\
{ }^{\circ} \mathrm{C}\end{array}$ & $\begin{array}{c}\text { Perdida de } \\
\text { masa, } \%\end{array}$ & $\begin{array}{c}\text { Efectos térmicos } \\
\text { de la temperatura } \\
\left({ }^{\circ} \mathrm{C} \text { ) (endo / exo) }\right. \\
\text { de la DTA }\end{array}$ \\
\hline Precursor 1 & 1 & $25-315$ & 19,6 & 160 (endo) \\
\hline & 2 & $315-660$ & 10,7 & 480 (exo) \\
\hline & 3 & $660-950$ & 9,9 & 905 (endo) \\
\hline Precursor 2 & 1 & $23-336$ & 19,3 & 170 e 191 (endo) \\
\hline & 2 & $336-642$ & 9,2 & 487 (exo) \\
\hline & 3 & $642-950$ & 9,7 & 927 (endo) \\
\hline Precursor 3 & 1 & $26-315$ & 18,3 & 165 (endo) \\
\hline & 2 & $315-635$ & 9,451 & 465 (exo) \\
\hline & 3 & $635-950$ & 11,0 & 908 (endo) \\
\hline
\end{tabular}

\section{Difractometria de Rayos - X (DRX)}

La Figura 2 nos presenta los difractogramas junto con el refinado por el método Rietveld referente a la síntesis del material cerámico en las tres condiciones distintas de síntesis, de acuerdo a lo presentado en Tabla 1.

La Figura 2 presenta los difractogramas referentes a las muestras del material $\left(\mathrm{SrCo}_{0,8} \mathrm{Fe}_{0,2} \mathrm{O}_{3-\delta}\right)$ obtenidas a partir de las condiciones experimentales $(1-3)$, de acuerdo a la Tabla 1.

Conforme a los resultados de las difratometria y refinado Rietveld presentados, la fase $\left(\mathrm{SrCo}_{0,8} \mathrm{Fe}_{0,2} \mathrm{O}_{3-\delta}\right)$ se obtuvo en todas las condiciones de síntesis adoptadas. Sin embargo, se observa una alteración en la cristalinidad del material obtenido a partir de los difractogramas cuando sustituimos uno de los reactivos adoptados en la síntesis; nitrato de Hierro III nanohidratado utilizado en la muestra 1 por el oxalato de Hierro III hexahidratado en la muestra 3. La utilización del nitrato de Hierro III nanohidratado en la muestra 1 presenta un material con mayor cristalinidad si lo comparamos con el resultado obtenido en la muestra 3, conforme Figura 2 (A) y (C), respectivamente. También se puede observar la diferencia de material de cristal de la intesidad de los polvos de difracción de la Figura $2(A)(B)$ y $(C)$ y sin embargo en la literatura. El material se obtiene por el método propuesto en el trabajo, polvos con una cristalinidad superior. 

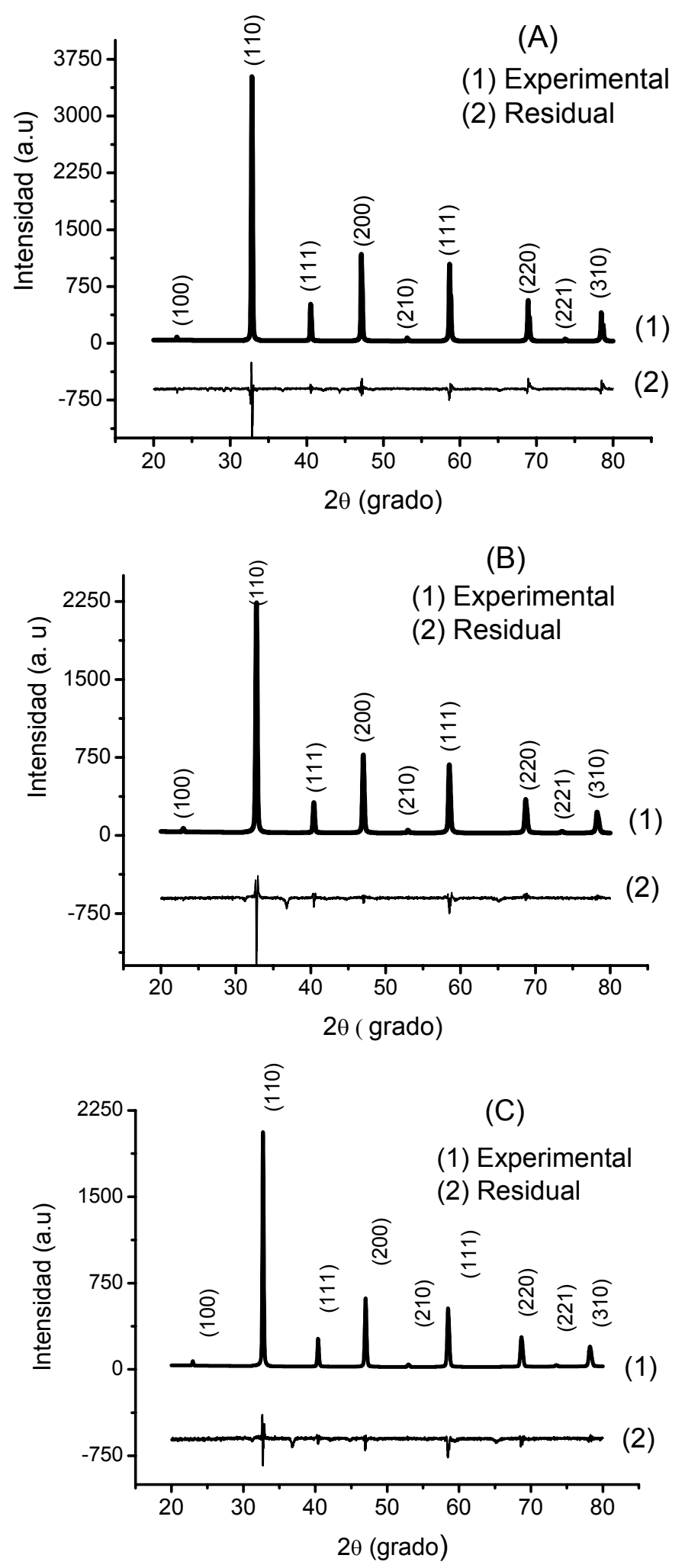

Fig. 2: Difratogramas junto con el refinado por el método Rietveld referente a la síntesis del material cerámico en las condiciones de síntesis: (A) Muestra 1, (B) Muestra 2 y (C) Muestra 3.

Tal comportamiento se puede atribuir a la diferencia de solubilidad existente entre los nitratos y los oxalatos; ya que los nitratos son completamente solubles en agua y los oxalatos no. Existe una situación más favorable para el crecimiento del tamaño mediano del cristalito y en su caso, de los nitratos, ya que siendo los nitratos más reactivos se dispone de un tiempo mayor para que el grano se desarrolle y generar consecuentemente granos con mayor tamaño medio de cristalito. A 
su vez, usando oxalatos, como son parcialmente solubles en agua, se genera en cierta forma una resistencia a la reacción de modo que será necesario un tiempo mayor de reacción al no tener el grano condiciones propicias para desarrollarse y, por lo tanto, se formaran cristalitos de menor tamaño medio.

El uso de nitrato de metales propicia mejores condiciones de síntesis, pues tienen una cinética de descomposición más favorable comparados a los carbonatos correspondientes. Esto lleva a una reducción de temperatura de calcinación.

Según los difractogramas presentados en la Figura $2(B)$ y $(C)$, se puede observar que el agente precipitante adoptado en la síntesis prácticamente no influyó en la formación de la fase $\mathrm{SrCo}_{0,8} \mathrm{Fe}_{0,2} \mathrm{O}_{3-\delta}$. Se percibe además, a partir de los resultados presentados en los difratogramas una pequeña variación en la distancia interplanar entre los datos experimentales y la carta patrón (Harrison et al., 1995), atribuida a condiciones experimentales distintas.

La Tabla 2 presenta los parámetros cristalográficos de las muestras obtenidas a partir de las condiciones experimentales de síntesis adoptadas (1 - 3) obtenidos a partir de los difractogramas y del refinado por el método Rietveld.

Tabla 2. Parámetros cristalográficos de los materiales obtenidos a partir del refinado Rietveld

\begin{tabular}{|c|c|c|c|c|}
\hline Muestra & $\begin{array}{c}\text { Parámetros de red } \\
(\mathrm{a}=\mathrm{b}=\mathrm{c}), \AA\end{array}$ & $\begin{array}{c}\text { Densidad, } \\
\mathrm{g} \cdot \mathrm{cm}^{-3}\end{array}$ & $\begin{array}{c}\text { Tamaño medio } \\
\text { de cristalito, } \mathrm{nm}\end{array}$ & $\begin{array}{c}\text { Parámetro } \\
\text { Residual, S }\end{array}$ \\
\hline 1 & 3,84 & 5,55 & 77,22 & 1,32 \\
\hline 2 & 3,86 & 5,50 & 42,95 & 1,24 \\
\hline 3 & 3,86 & 5,49 & 42,13 & 1,21 \\
\hline
\end{tabular}

A partir de los resultados presentados en la Tabla 2 se puede verificar la variación en el tamaño mediano de cristalito en función de los parámetros de síntesis modificados. Cuando la síntesis se realiza con nitrato de Hierro III nanohidratado (muestra 1) el tamaño mediano de cristalito fue aproximadamente igual la $77,22 \mathrm{~nm}$, casi un $83 \%$ superior al obtenido usando oxalato de Hierro III $(42,23 \mathrm{~nm})$ y la mezcla equimolar de $\left(\mathrm{NH}_{4}\right)_{2} \mathrm{C}_{2} \mathrm{O}_{4} \mathrm{H}_{2} \mathrm{O}$ e $\mathrm{C}_{6} \mathrm{H}_{8} \mathrm{O}_{7}$ como agente precipitante en la muestra $3(42,23 \mathrm{~nm})$ y en la muestra $2(42,95 \mathrm{~nm})$ utilizando sólo el $\left(\mathrm{NH}_{4}\right)_{2} \mathrm{C}_{2} \mathrm{O}_{4} \mathrm{H}_{2} \mathrm{O}$ como agente precipitante. Este resultado está de acuerdo con los difractogramas presentados en la Figura 2 (A) y (C), es decir, en las condiciones de muestra 1, que presentó mayor cristalinidad, también se obtuvo mayor tamaño mediano de cristalito. La diferencia de aproximadamente el $83 \%$ del tamaño medio de cristalito entre las muestras 1 y 3 se debe probablemente a la diferencia entre las solubilidades del nitrato y oxalato de hierro (III) que influyen en la nucleación de los granos durante el proceso de síntesis.

Situados ya en el caso donde hubo cambios en el agente precipitante adoptado en la síntesis, el tamaño mediano del cristalito prácticamente no varió $(42,95 \mathrm{~nm}$ y $42,23 \mathrm{~nm})$, conforme a las muestras 2 y 3 , respectivamente, si los comparamos con los reactivos de la misma naturaleza.

El análisis de los párámetros de red y la densidad presentado confirman en el Cuadro 2, confirmó la estructura cúbica del material obtenido por el método de coprecipetación a través de oxalato y refinamiento por el método de Rietveld, estos resultados cooperando con los citados por Harrison et al., 1995 (parámetros de red: $a=b=c=3,8491$ y la densidad $=5,55$ ). El ajuste obtenido por los refinados realizados se puede confirmar con el valor encontrado para el parámetro residual, $S$ muy cerca de la unidad. Por lo tanto, los valores obtenidos por el refinado Rietveld se consideran fiables.

Conforme a los resultados de difractometria y refinamiento Rietveld presentados, la fase $\left(\mathrm{SrCo}_{0,8} \mathrm{Fe}_{0,2} \mathrm{O}_{3-\delta}\right)$ fue obtenida en todas las condiciones de síntesis. Sin embargo, se observa que la cristalinidad y el tamaño medio de cristalito del material son afectados de acuerdo con las alteraciones realizadas, sea en el agente precipitante o en la naturaleza del reactivo adoptado. 
La síntesis con base en el método vía co-precipitación en medio oxalato nos permitió obtener granos con una franja medía de tamaño medio de cristalito de entre aproximadamente 42 y $77 \mathrm{~nm}$, conforme las condiciones de síntesis empleadas. Tales resultados se compararon con los obtenidos a partir del método de creación de complejo combinado EDTA - citrato, adoptando las mismas condiciones en el tratamiento térmico. En el caso, de la síntesis realizada por este último método obtuvimos granos con tamaño mediano de cristalito en la franja de los $45 \mathrm{~nm}$, mismo orden de magnitud que los obtenidos por el método vía co-precipitación en medio oxalato.

\section{Microscopía electrónica de barrido (MEV)}

La Figura 3 presenta el resultado del análisis de microscopía electrónica de barrido (MEV) del material $\left(\mathrm{SrCo}_{0,8} \mathrm{Fe}_{0,2} \mathrm{O}_{3-\delta}\right)$ obtenidos a partir de las condiciones de síntesis $(1-3)$, como se muestra en la Tabla 1. La micrografia del material se sometió a una ampliación igaul a 5000 x.
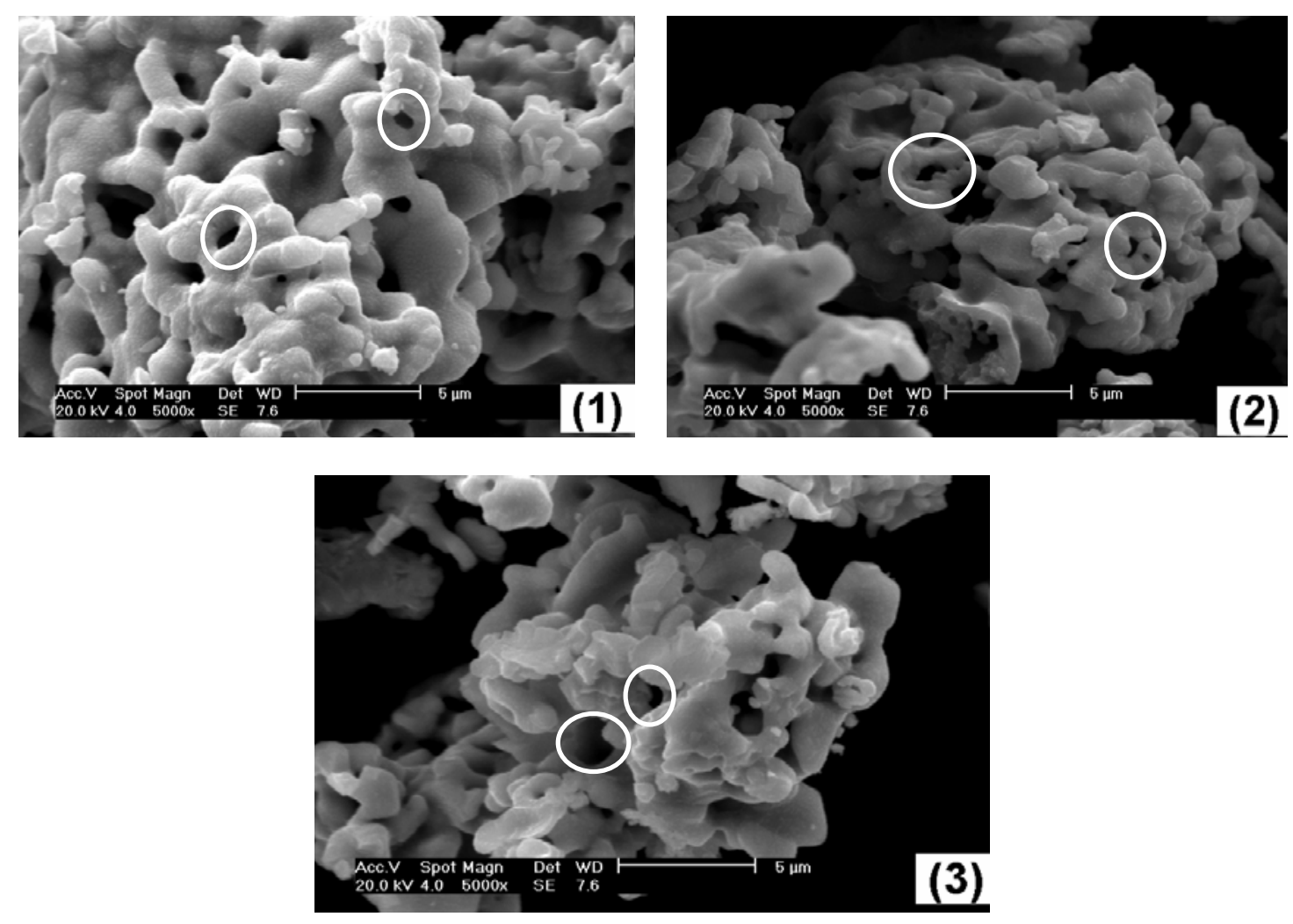

Fig. 3: microcospia electrónica de barrido del material $\left(\mathrm{SrCo}_{0,8} \mathrm{Fe}_{0,2} \mathrm{O}_{3-\delta}\right)$ obtenidos a partir de las condiciones de síntesis (1 - 3). Aplicación: 5000 x.

De la Figura 3 se puede observar el tamaño de las partículas no es uniforme, con una muestra compuesta de partículas o grupos de particulas, teniendo en cuenta también la presencia de poros, como se destaca en la Figura 3. En cooperación con los resultados presentados por Rui et al., 2010.

\section{Adsorción de nitrógeno (Método de BET)}

Las medidas propiedades textuais fueron tomadas de la muestra es representativa de 3 muestras de otra, con superficie específica y volumen de poros igual a la aproximadamente $4,41 \mathrm{~m}^{2} \mathrm{~g}^{-1} \mathrm{y}$ $0,009 \mathrm{~cm}^{3} \mathrm{~g}^{-1}$, respectivamente. Sin embargo, el material $\left(\mathrm{SrCo}_{0,8} \mathrm{Fe}_{0,2} \mathrm{O}_{3-\delta}\right)$ muestra la superficie en el mismo orden de magnitud que el apresentado por Rui et al., $2010\left(6,61 \mathrm{~m}^{2} \mathrm{~g}^{-1}\right)$, sino de un método de síntesis diferentes (métodos el citrato) y se calcina a una temperatura $\left(900{ }^{\circ} \mathrm{C}\right)$, también es diferente de la utilizada en el método de síntesis de este estudio $\left(950{ }^{\circ} \mathrm{C}\right)$. Esta 
variación existe en la superficie puede justificarse por la diferencia de temperatura de calcinación, aprobada por los autores y utiliza en este trabajo, ya que el aumento de la temperatura conduce a una sinterización de las partículas como también el uso de diferentes de síntesis.

La Figura 4 muestra las isotermas de fisisocrión de nitrógeno para el material $\left(\mathrm{SrCo}_{0,8} \mathrm{Fe}_{0,2} \mathrm{O}_{3-\delta}\right)$ sintetizados y calcinados.

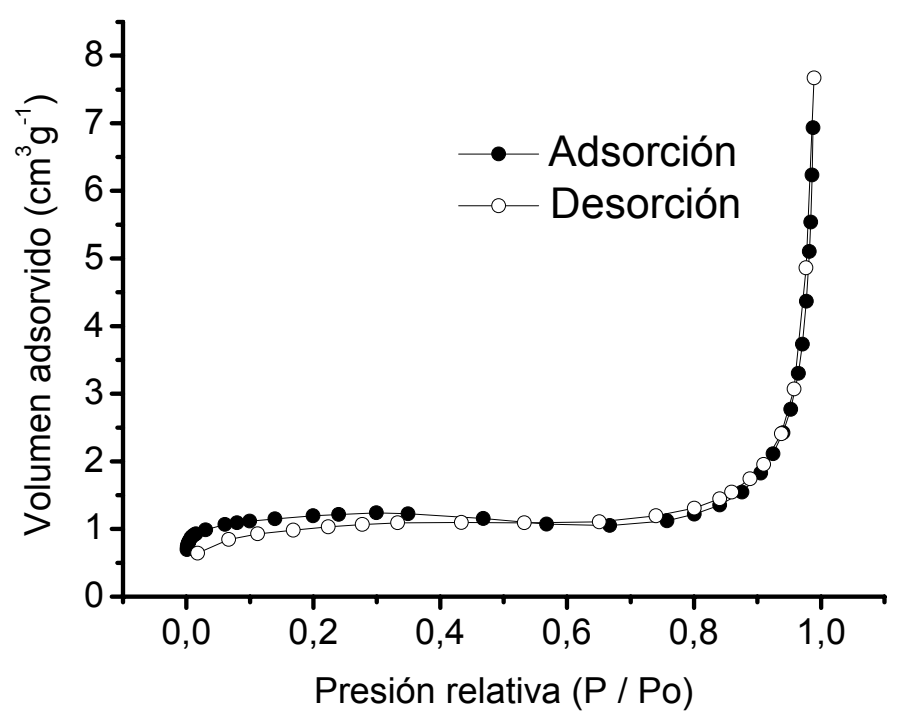

Fig. 4: Análisis de la adsorción / desorción del material de nitrógeno $\left(\mathrm{SrCo}_{0,8} \mathrm{Fe}_{0,2} \mathrm{O}_{3-\delta}\right)$.

Como se muestra en la Figura 4 muestran isotermas del tipo II (Ciola, 1981). Este tipo de isotermas se ecuentra cuando se produce la adsorción sobre materiales no porosos poros de gran diámetro. Las isotermas presentan dos regiones distintas, el proceso de adsoción monocopa se produce a baja presión, seguido de la rodilla o el punto de inflexión correspondientes para completar la cobertura de la primera capa. Con la creciente presión sobre el sólido está completamente cubierto con varias capas que ocurren en la infinidad de saturación enfoques.

\section{CONCLUSIONES}

A partir de los análisis TG / DTA presentados podemos observar las tres pérdidas de masa durante las etapas de calentamiento y el mecanismo de reacción propuesto para la obtención de la fase perovsquita $\mathrm{SrCo}_{0,8} \mathrm{Fe}_{0,2} \mathrm{O}_{3-\delta}$. A partir del refinado por el método Rietveld se puede verificar el tamaño medio del cristalito del material obtenido en las distintas condiciones de síntesis adoptada. Cuando la síntesis fue realizada con nitrato de Hierro III nanohidratado, el tamaño mediano del cristalito fue cerca de $77,22 \mathrm{~nm}$, casi un $83 \%$ superior al obtenido usando oxalato de Hierro III $(42,23 \mathrm{~nm})$, mientras que la variación del agente precipitante prácticamente no afectó al tamaño medio de cristalito $(42,95 \mathrm{~nm}$ y $42,23 \mathrm{~nm})$ y la mezcla equimolar de $\left(\mathrm{NH}_{4}\right)_{2} \mathrm{C}_{2} \mathrm{O}_{4} \mathrm{H}_{2} \mathrm{O}$ y $\mathrm{C}_{6} \mathrm{H}_{8} \mathrm{O}_{7}$ como agente precipitante.

El material presenta tamaño de las partículas no es uniforme y isotermas de tipo II, con superficie especifica y volumen de poros iagual a aproximadamente $4,41 \mathrm{~m}^{2} \mathrm{~g}^{-1}$ y $0,009 \mathrm{~cm}^{3} \mathrm{~g}^{-1}$, respectivamente.

\section{AGRADECIMIENTOS}

Al CNPQ/CT-[Energ]/CT-Petro, la [UFRN]/[PPGEQ]/[LTR], al [NEPGN] y Universidad del Sur Toulon - Var, IM2NP - UMR CNRS 6242. 


\section{REFERENCIAS}

Casanova J. R., Kharton V. V. y Marques F. M. B., Obtención y Caracterización de Muestras Cerâmicas de $\mathrm{La}_{(1-x)} \mathrm{Sr}_{x} \mathrm{Ga}_{(1-x)} \mathrm{Fe}_{x} \mathrm{O}_{3}$, Revista Matéria, 9, 222-226 (2004).

Ciola, R. Fundamentos da catálise, São Paulo: Ed. Moderna: Ed. da universidade de São Paulo, 59, (1981).

Dong $\mathrm{H}$. y otros cinco autores, Investigation on POM reaction in a new perovskite membrane reactor. Catalysis Today, 67, 3-13 (2001).

Gualtieri M. L., Prudenziati M., Gualtieri A. F., Quantitative determination of the amorphous phase in plasma sprayed alumina coatings using the Rietveld method, Surface \& Coatings Technolog, 201, 2984-2989 (2006).

Harrison, W.T.A. y otros cinco autores, A neutron diffraction study of two strontium cobalt iron oxides, Journal of Materials Research Bulletin, 30, 621-630 (1995).

Ikeguche M., y otros cuatro autores, Reaction and oxygen permeation studies in $\mathrm{Sm}_{0.4} \mathrm{Ba}_{0.6} \mathrm{Fe}_{0.8} \mathrm{Co}_{0.2} \mathrm{O}_{3-\delta}$ membrane reactor for partial oxidation of methane to syngas, Applied Catalysis A: General, 290, 212-220 (2005).

Ikeguchi M. y y otros cinco autores, Effects of preparation method on oxygen permeation properties of $\mathrm{SrFeCO} \mathrm{C}_{0.5} \mathrm{O}_{x}$ membrane, Separação e Purificação Tecnologia, 32, 313-318 (2003).

Liu W. y otros cinco autores, Electrical conduction and oxygen transport in SrFeCoO oxide, Membranes Solid State Ionics, 135, 727-730 (2000).

$\mathrm{Lu} \mathrm{H}$. y otros tres autores, Partial oxidation of methane in $\mathrm{Ba}_{0.5} \mathrm{Sr}_{0.5} \mathrm{Co}_{0.8} \mathrm{Fe}_{0.2} \mathrm{O}_{3-\delta}$ membrane reactor at high pressures, Catalysis Today, 104, 154-159 (2005).

Lu H., Cong Y. y Yang W.S., Oxygen permeability and stability of $\mathrm{Ba}_{0.5} \mathrm{Sr}_{0.5} \mathrm{Co}_{0.8} \mathrm{Fe}_{0.2} \mathrm{O}_{3^{-}}{ }_{\delta}$ as an oxygen-permeable membrane at high pressures, Solid State lonics, 177, 595-600 (2006).

Martínez J.R. y otros cuatro autores, Rietveld refinement of amorphous SiO 2 prepared via sol-gel method, Materials Letters, 60, 3526-3529 (2006).

Mcintosh S. y otros siete autores, Phase stability and oxygen non-stoichiometry of $\mathrm{SrCO}_{0.8} \mathrm{Fe}_{0.2} \mathrm{O}_{3-\delta}$ measured by in situ neutron diffraction, Solid State lonics, 177, 833-842 (2006).

Mineshige A. I. y otros cinco autores, Introduction of A-site deficiency into $\mathrm{La}_{0.6} \mathrm{Sr}_{0.4} \mathrm{Co}_{0.2} \mathrm{Fe}_{0.8} \mathrm{O}_{3-\delta \text {, }}$ Solid State lonics, 176, 1145-1149 (2005).

Oliveira A. P. A. De y otros cuatro autores, Synthesis of $\mathrm{BaCeO}_{3}$ and $\mathrm{BaCe}_{0.9} \mathrm{Y}_{0.1} \mathrm{O}_{3-\delta}$ from mixed oxalate precursors, Journal of the European Ceramic Society, 27, 3597-3600 (2007).

Ouaguenouni M. H y otros tres autores, Preparation and catalytic activity of nickelemanganese oxide catalysts in the reaction of partial oxidation of methane, Comptes Rendus Chimie, 12, 740747 (2009).

Qui L., y otros cuatro autores, Oxygen permeation studies of $\mathrm{SrCo}_{0.8} \mathrm{Fe}_{0.2} \mathrm{O}_{3-}$, Solid State Ionics, 76, 321-329 (1995).

Rivas M. E., y otros seis autores, Structural features and performance of $L a N i_{1}{ }_{x} R h_{x} O_{3}$ system for the dry reforming of methane, Applied Catalysis A: General, 344, 10-19 (2008). 
Rui Z. y otros tres autores, $\mathrm{SrCo}_{0.8} \mathrm{Fe}_{0.2} \mathrm{O}_{-\delta}$ sorbent for high-temperature production of oxygenenriched carbon dioxide stream, Fuel, 89, 1429-1434 (2010).

Shao Z. y otros cinco autores, Investigation of the permeation behavior and stability of a $\mathrm{Ba}_{0.5} \mathrm{Sr}_{0.5} \mathrm{Co}_{0.8} \mathrm{Fe}_{0.2} \mathrm{O}_{3-\delta \delta}$, oxygen membrane, Journal of Membrane Science, 172, 177-188 (2000).

Shao Z. y otros cuatro autores, Performance of a mixed-conducting ceramic membrane reactor with high oxygen permeability for methane conversion, Journal of Membrane Science, 183, 181192 (2001).

Snyder, R.L., The Use of Reference Intensity Ratios in X-Ray Quantitative Analysis, Pow. Diff, 186-193 (1992).

Tanabe Y. E. y Assaf E. M., Óxidos do tipo perovskita para reação de redução de NO Com CO, Química Nova, 32, 1129-1133 (2009).

Teraoka Y. y otros cuatro autores, Catalytic effects in oxygen permeation through mixedconductive LSCF perovskite membranes, Solid State lonics, 152-153, 681-687 (2002).

Teraoka Y., Nobunaga T. y Yamozoe N., Effect of cation substitution on the oxygen semipermeability of perovskite-type oxides, Chemical Letters, 503-506 (1988).

Tong J. y otros tres autores, Novel and ideal zirconium-based dense membrane reactors for partial oxidation of methane to syngas. Catalysis Letters, 78, 129-137 (2002).

Vargas R.A., y otros tres autores, Síntese e Caracterização dos Pós de $\mathrm{Nd}_{1-x} \mathrm{Sr}_{x} \mathrm{MnO}_{3}$ e $\mathrm{La}_{1-}$ ${ }_{x} \mathrm{Sr}_{x} \mathrm{Co}_{1-y} \mathrm{Fe}_{y} \mathrm{O}_{3}$, Revista Matéria, 12, 8-21 (2007).

Waller D., y otros tres autores, The effect of thermal treatment on the resistance of LSCF electrodes on gadolinia doped ceria electrolytes, Solid State Ionics, 86-88, 767-772 (1996).

Wilson A. J. C, International tables for crystallography: mathematical, physical and chemical tables. 2ed. Dardrecht: klerwer Academic Publishers, v. C, cap. 8 e 6, 625-626 (1995).

Yang L. y otros cinco autores, Role of $\mathrm{ZrO}_{2}$ addition on oxygen transport and stability of $\mathrm{ZrO}_{2}-$ promoted $\mathrm{SrCO}_{0.4} \mathrm{Fe}_{0.6} \mathrm{O}_{3-\delta}$, Separation and Purification Technology, 32, 301-306 (2003).

Yaremchenlo A.A. y Kharton V.V., Oxygen permeability of perovskite type $B a B i_{1-x} L a_{x} O_{3-\delta}$, Mater. Res. Bull. 33, 1027 - (1999).

Young R. A., The Rietveld Method .I.U.Cr. New York, Oxford University Press Inc. (1995). 